
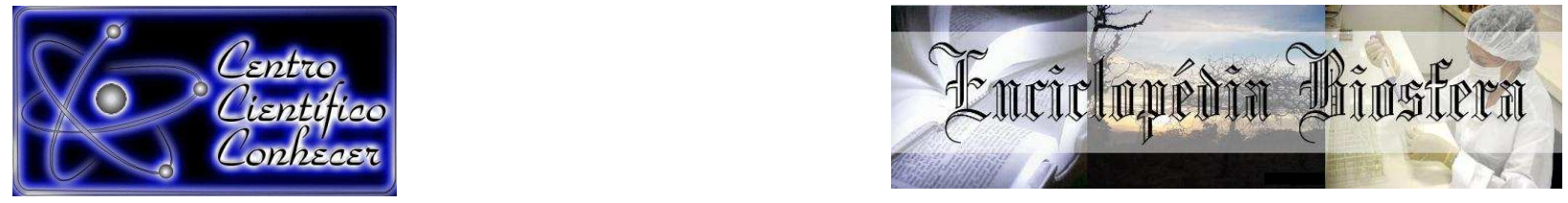

\title{
COMERCIALIZAÇÃO DE HORTIGRANJEIROS NA REGIÃO SUL DA CEASA/ES DURANTE O PERÍODO DE 2005-2012
}

\author{
Wellington Abeldt Erlacher ${ }^{(1)}$, Fábio Luiz de Oliveira ${ }^{(2)}$ Alisson Moura de Souza ${ }^{(3)}$, \\ Gisele Rodrigues Moreira ${ }^{(2)}$, Diego Mathias Natal da Silva ${ }^{(4)}$ \\ (1) Graduando em Agronomia, Centro de Ciências Agrárias da Universidade Federal \\ do Espírito Santo (CCA-UFES) (wellington_abeldt@hotmail.com); \\ (2) Professores da Universidade Federal do Espírito Santo, Centro de Ciências \\ Agrárias da Universidade Federal do Espírito Santo (CCA-UFES); \\ (3) Agrônomo, Centro de Ciências Agrárias da Universidade Federal do Espírito \\ Santo (CCA-UFES); \\ (4) Pós-Graduando em Produção Vegetal, Centro de Ciências Agrárias da \\ Universidade Federal do Espírito Santo (CCA-UFES). Alegre - Brasil.
}

Recebido em: 08/09/2015 - Aprovado em: 14/11/2015 - Publicado em: 01/12/2015 DOI: http://dx.doi.org/10.18677/Enciclopedia_Biosfera_2015_135

Os objetivos desse trabalho foram conhecer a contribuição dos estados e municípios para o abastecimento da Regional Sul da CEASA/ES, quanto à quantidade e tipos de hortaliças e frutas comercializadas, além de delinear os possíveis fatores que determinam a distribuição nesse mercado, apontando os espaços que são ocupados por municípios de outras regiões do estado e até por outros estados, de modo a servir como orientação aos produtores da região visando à ocupação de mercado. Todos os dados foram coletados na CEASA/ES-Sul localizada na rodovia Mauro Miranda S/N, Bairro Valão, na cidade de Cachoeiro de Itapemirim/ES. A coleta dos dados referentes a: tipo, origem, quantidades e destinatários de cada produto, foi realizada através de um formulário chamado "romaneio" de mercadoria, que é entregue pelo condutor da mercadoria (motorista do caminhão ou o próprio agricultor), ou anotado por um funcionário da CEASA/ES-SUL, na portaria central no momento da entrada das mercadorias. Os resultados mostram um valor crescente de produtos comercializado e receitas na CEASA/ES-Sul entre os anos de 2005 a 2012, sendo que o volume comercializado em 2005 foi de 16,7 mil toneladas e em 2012 foi de 32 mil toneladas. Os produtores do sul do Espírito Santo e regiões e circunvizinhas, ocuparam apenas 43 e $41 \%$ do mercado para as frutas e as hortaliças de frutas no ano de 2012, respectivamente. E em torno dos 30 a $38 \%$ do mercado das hortaliças de tubérculos, folhosas e raízes. Isso aponta para as demandas de mercado para esses produtos, demonstrando oportunidade de produção regional de hortifrutigranjeiros para ocupação desse mercado.

PALAVRAS-CHAVE: Centrais de abastecimento, hortaliças, mercado. 


\title{
ASSOCIATED TO OLERICULTURE OF MERCHANTABILITY OF DIAGNOSIS THE SOUTH REGION OF CEASA / ES
}

\begin{abstract}
The objectives of this study is to evaluate the contribution of states and municipalities to supply the Southern Regional CEASA/ES, as to the amount and types of vegetables marketed and outlines the possible factors that determine the distribution in this market, pointing spaces that are occupied by municipalities in other regions of the state and even other states, to serve as guidance to producers in the region to the occupation market. All data were collected in the CEASA / ES-South localized on the highway Mauro Miranda S / N, Walloon district in the city Cachoeiro de Itapemirim / ES. The gathering of data on type, origin, quantities and recipients of each product was conducted through a form called "your packing list" merchandise that is delivered by the driver of the goods (truck driver or the farmer himself), or annotated per an official from CEASA / ES-south, in Central ordinance the time of entry of the goods. The results show a growing value of marketed products and recipes in the CEASA / ES-south between the years 2005-2012, and the traded volume in 2005 was 16.7 thousand tons and in 2012 was 32 thousand tons. The producers of the south of the Holy Spirit and regions and surrounding occupied only 43 and $41 \%$ of the market for fruit and vegetable crops fruit in 2012 , respectively. This points to the market demand for those products, demonstrating regional of production of hortifrutigranjeiros opportunity for occupancy this market.
\end{abstract}

KEYWORDS: Supply centers, vegetables, market.

\section{INTRODUÇÃO}

O sistema das Centrais de Abastecimento (CEASA's) surgiu com o intuito de atuar no mercado como entreposto comercial capaz de organizar a comercialização desde a produção até a distribuição varejista, confeccionando uma interação entre produção e comercialização capaz de minimizar os efeitos negativos da intermediação (AZEVEDO \& FAULIN, 2003). A CEASA tem função central de distribuição, sendo importante para a redução dos custos e aumentar a qualidade dos produtos hortifrutigranjeiros em cada estado (FARIA \& SOUSA, 2014).

As facilidades oferecidas por esses espaços é a redução de gastos no transporte e diminuição de perdas (pela possibilidade de estocagem), além da agilidade na comercialização, pois é o ponto de reunião de todos os agentes da cadeia produtiva (CUNHA \& CAMPOS, 2008). A infraestrutura das CEASA's colabora para que esses se tornassem locais de referência para a comercialização dos produtos hortifrutigranjeiros. Normalmente se tem estrutura de todos os aspectos, desde o setor de insumos até o financeiro, que facilitam a realização do negócio (CUNHA, 2006).

Neste sentido é nítida a importância e evolução dos produtos hortigranjeiros no cenário nacional (CUNHA \& BELIK, 2012). Tanta importância e crescimento de produtos hortigranjeiros que no ano de 2001 foi criada a CEASA/ES-Sul (Centrais de Abastecimento do Espírito Santo, Regional Sul) na cidade de Cachoeiro de Itapemirim/ES, que contava apenas com o galpão não permanente, conhecido como o galpão da "pedra", espaço onde ocorrem as negociações no varejo do produtor diretamente com o consumidor. Em 2003 a CEASA/ES-Sul foi reestruturada agregando os galpões que abrigam as 16 lojas atacadistas, que realizam a comercialização no âmbito da revenda, intermediando a relação entre o produtor e o consumidor. 
Desde a criação da CEASA/ES-Sul não foram realizados estudos que demonstrassem a real influência desse canal na comercialização de hortigranjeiros na região Sul do Espírito Santo, no que diz respeito à origem dos produtos comercializados e os espaços que são ocupados por municípios de outras regiões do estado e até por outros estados.

Neste contexto, os objetivos desse trabalho foram conhecer a contribuição dos estados e municípios para o abastecimento da CEASA/ES-Sul, quanto à quantidade e tipos de hortaliças e frutas comercializadas, além de delinear os possíveis fatores que determinam a distribuição nesse mercado, apontando os espaços que são ocupados por municípios de outras regiões do estado e até por outros estados, de modo a servir como orientação aos produtores da região visando à ocupação de mercado.

\section{MATERIAL E MÉTODOS}

Para a realização deste trabalho, optou-se por um estudo exploratório pelo fato do mesmo permitir que o investigador aumente sua experiência em torno do problema pesquisado, dentro dos limites de uma realidade específica, buscando antecedente e maior conhecimento sobre o assunto (PIOVESAN \& TEMPORINI, 1995).

Todos os dados foram coletados na CEASA/ES-Sul localizada na rodovia Mauro Miranda S/N, Bairro Valão, na cidade de Cachoeiro de Itapemirim/ES. A coleta dos dados referentes a: tipo, origem, quantidades e destinatários de cada produto, foi realizada através de um formulário chamado "romaneio" de mercadoria, que é entregue pelo condutor da mercadoria (motorista do caminhão ou o próprio agricultor), ou anotado por um funcionário da CEASA/ES-SUL, na portaria central no momento da entrada das mercadorias.

Durante o ano de 2012 os romaneios foram coletados diariamente e as informações sistematizadas e processadas. Também foi observado o valor médio pago por cada classe de produto diariamente, para que fosse calculada a receita gerada, no geral e por classe de produto. As informações anteriores a 2012 foram obtidas do banco de dados histórico da administração da CEASA/ES-Sul, que oferecia dados a partir de 2005.

A partir da coleta dessas informações foi possível determinar o volume comercializado, contribuição dos diferentes estados e municípios,, no geral e por classe de hortifrutigranjeiros. Os dados coletados foram analisados por meio de estatística descritiva, com base em tabelas e gráficos. A classificação dos hortigranjeiros foi realizada segundo FILGUEIRA (2008).

\section{RESULTADOS E DISCUSSÃO}

Os resultados mostram um valor crescente de produtos comercializado e receitas na CEASA/ES-Sul entre os anos de 2005 a 2012, sendo que o volume comercializado em 2005 foi de 16,7 mil toneladas e em 2012 foi de 32 mil toneladas. Já a receita seguiu o mesmo padrão do volume comercializado, aumentando de $R \$$ 20,8 milhões de Reais em 2005 para $R \$$ 45,4 milhões de Reais em 2012 (Figura 1). 


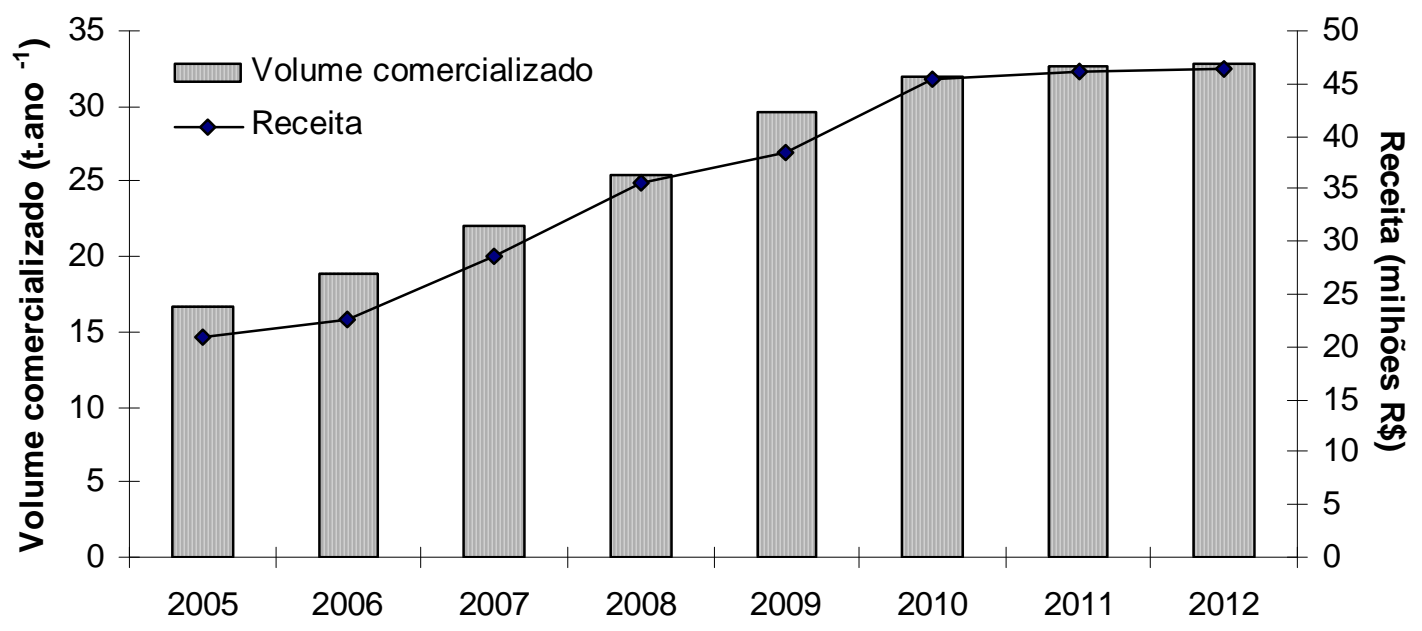

FIGURA 1- Volume comercializado e receita gerada com hortigranjeiros na CEASA/ES-Sul no período de 2005 a 2012. Cachoeiro de Itapemirim/ES, CCA/UFES

Com o aumento da movimentação de produtos e receitas nos últimos anos na CEASA/ES-Sul, é notória a importância da CEASA/ES-Sul para a comercialização dos produtos no sul do estado do Espírito Santo, facilitando a cadeia de abastecimento regional, encurtando o caminho entre 0 produtor e 0 consumidor final, assim como observado por FARIA \& SOUSA (2014) em Minas Gerais e CUNHA \& BELIK (2012) nas principais centrais de abastecimento do Brasil no período de 2000 a 2009.

Esse canal tem atraído as redes de comercialização local de todos os portes, desde pequenos comerciantes de hortifrutigranjeiros até os grandes supermercados, o que reduz o preço final para o consumidor, além de aumentar as vendas do produtor, o que possibilita maiores investimentos e melhorias na qualidade produtiva.

Todavia é importante ressaltar que em 2011 e 2012, foi observada estabilidade no volume comercializado de 32,8 mil toneladas. Tal fato pode ser justificado pela limitação no espaço físico da CEASA/ES-Sul que se encontra em capacidade máxima de trabalho, visto que as 16 lojas do galpão atacadista estão ocupadas e os 40 pontos do galpão do produtor ("pedra"). Neste contexto, a ampliação do espaço físico da CEASA/ES-Sul, é de extrema valia para o crescimento desse canal de comercialização. De acordo com WEGNER \& BELIK (2012) os principais problemas para o funcionamento das CEASAS é a falta de gestão e recursos financeiros, que dificulta o papel das centrais na distribuição e abastecimento.

Salienta-se que de todos os produtos comercializados na CEASA/ES-Sul no ano de $2012,78,9 \%$ do volume comercializado (25,2 mil toneladas) e $75,15 \%$ da receita ( $R$ \$ 34,1 milhões de reais), são provenientes do estado do Espírito Santo, o restante vem de outros estados (Tabela 1). Esses resultados mostram novamente a importância da CEASA/ES-Sul, para os municípios do estado, sendo também uma importante geradora de divisas para o próprio estado e em especial para a região Sul do Espírito Santo. 
TABELA 1- Contribuição dos diferentes estados no volume comercializado e na receita gerada com hortigranjeiros na CEASA/ES-Sul no ano de 2012. Cachoeiro de Itapemirim/ES, CCA/UFES.

\begin{tabular}{ccccc}
\hline Estados & Toneladas & $\%$ & Receita $(\mathrm{R} \$)$ & $\%$ \\
\hline Espírito Santo & $25.251,35$ & 78,92 & $34.134 .515,23$ & 75,15 \\
Minas Gerais & $2.636,37$ & 8,23 & $4.301 .687,94$ & 9,47 \\
São Paulo & $1.264,77$ & 3,95 & $1.975 .794,97$ & 4,35 \\
Rio Grande do Sul & 816,84 & 2,55 & $1.784 .159,61$ & 3,92 \\
Bahia & 577,48 & 1,80 & $582.613,94$ & 1,28 \\
Santa Catarina & 552,84 & 1,72 & $944.966,50$ & 2,08 \\
Outros & 896,73 & 2,80 & $1.692 .324,81$ & 3,72 \\
\hline Total & $31.996,38$ & 100 & $45.416 .063,00$ & 100 \\
\hline
\end{tabular}

Toda a contribuição do estado do Espírito Santo no volume comercializado no ano de 2012 (50,95\%) foram fornecidos pela CEASA/ES (Cariacica), 20,59\% pelo município de Venda Nova, 9,94\% por Vargem alta, 8,82\% por Cachoeiro de Itapemirim, 3,09\% por Atílio Vivacqua e 2,59\% por Iconha, o restante é representado pelos demais munícipios do estado. A receita gerada segue a mesma tendência do volume comercializado (Tabela 2).

Os resultados mostram que a CEASA/ES (Cariacica) é responsável pela metade dos produtos comercializados, denotando que ainda existe espaço para a participação de agricultores dos municípios do Sul do estado e regiões circunvizinhas, no canal de comercialização na CEASA/ES-Sul. Segundo COSER et al. (2011), a maior parte dos produtos hortifrutigranjeiros comercializados em Erechim-RS são produzidos em outras regiões, vindo principalmente de outras CEASAS, e apenas uma pequena parte é proveniente do munícipio, ressalta-se que os principais fatores que levam á aquisição de produtos de outros municípios são a carência de conhecimento técnicos dos agricultores associada a baixa qualidade dos produtos e instabilidade de produção.

TABELA 2- Contribuição dos diferentes municípios do Espírito Santo no volume comercializado e na receita gerada com hortigranjeiros na CEASA/ES-Sul no ano de 2012. Cachoeiro de Itapemirim/ES, CCA/UFES.

\begin{tabular}{ccccc}
\hline Municípios & Toneladas & $\%$ & Valor $(\mathrm{R} \$)$ & $\%$ \\
\hline $\begin{array}{c}\text { Cariacica (Ceasa } \\
\text { Central) }\end{array}$ & $12.866,62$ & 50,95 & $21.114 .146,47$ & 61,85 \\
Venda Nova do & $5.199,94$ & 20,59 & $5.626 .422,99$ & 16,48 \\
$\quad$ Imigrante & & & & \\
Vargem Alta & $2.511,41$ & 9,94 & $2.504 .369,26$ & 7,33 \\
Cachoeiro do Itapemirim & $2.228,50$ & 8,82 & $2.029 .305,08$ & 5,94 \\
Atílio Vivacqua & 782,22 & 3,09 & $613.302,90$ & 1,79 \\
Iconha & 655,38 & 2,59 & $652.338,52$ & 1,91 \\
Outros & $1.007,25$ & 3,98 & $1.594 .630,01$ & 4,67 \\
\hline Total & $25.251,32$ & 100 & $34.134 .515,23$ & 100 \\
\hline
\end{tabular}

Os produtos comercializados por classes hortigranjeiras mostram que a maior parte do volume são as frutas $(57,01 \%)$, hortaliça de fruto $(59,01 \%)$, hortaliça ENCICLOPÉDIA BIOSFERA, Centro Científico Conhecer - Goiânia, v.11 n.22; p. 971 
tubérculo $(66,64 \%)$, hortaliças folhosas $(61,21 \%)$, hortaliças de raiz $(69,45)$, hortaliças de bulbo $(83,15 \%)$ vêm de outras regiões do estado ou de outros estados, com exceção da hortaliça de flor, sendo $97,64 \%$ do volume comercializado proveniente do sul do estado do Espírito Santo (Tabela 3).

Esses resultados abrem a expectativa de espaço no mercado, que pode ser ocupada por produtores da região Sul do Espírito Santo e circunvizinhança, principalmente pelas hortaliças de fruto, folhosas e raízes. Existem várias espécies dentro desses grupos de hortaliças com aptidão ao cultivo na região, daí a necessidade de observar quais delas há demanda a serem supridas. Além disso, a inserção de novos hortigranjeiros no sul do estado ampliaria a mão de obra e receita regional, tendo novos investimentos municipais e como consequência 0 desenvolvimento regional.

TABELA 3 - Volume comercializado, por classe de hortigranjeiros, na CEASA/ES-Sul, procedentes da Região Sul do Espírito Santo e de outras regiões do Estado e de outros Estados, no ano de 2012. Cachoeiro de Itapemirim/ES, CCA/UFES.

\begin{tabular}{lcccc}
\hline \multirow{2}{*}{ Hortigranjeiros } & \multicolumn{2}{c}{ Sul do Espírito Santo } & \multicolumn{2}{c}{$\begin{array}{c}\text { Outras regiões do ES e } \\
\text { outros estados }\end{array}$} \\
\cline { 2 - 5 } & Toneladas & $\%$ & Toneladas & $\%$ \\
\hline Frutas & $3.824,60$ & 42,99 & $5.071,90$ & 57,01 \\
Hortaliças fruto & $3.799,82$ & 40,99 & $5.470,64$ & 59,01 \\
$\begin{array}{l}\text { Hortaliças } \\
\text { tubérculo }\end{array}$ & $1.463,13$ & 33,36 & $2.922,57$ & 66,64 \\
Hortaliças & $1.417,51$ & 38,79 & $2.236,77$ & 61,21 \\
folhosas & 882,44 & 30,55 & $2.006,35$ & 69,45 \\
Hortaliças raiz & 302,55 & 97,64 & 7,31 & 2,36 \\
Hortaliças flor & 292,52 & 16,85 & $1.443,51$ & 83,15 \\
Hortaliças bulbo & 83,69 & 61,38 & 52,66 & 38,62 \\
Outros & & & & \\
\hline
\end{tabular}

Das frutas (Banana, Laranja, Tangerina, Mamão, Manga e Goiaba) comercializadas na CEASA/ES-Sul no ano de 2012, com exceção da banana a demais frutas são provenientes de municípios de outras regiões do estado (Figura 2). De modo geral observa-se que na CEASA/ES-Sul as frutas com maior volume de comercialização é a banana seguida da laranja, diferente do que ocorre na CEASA do Rio Grande do Norte, onde as frutas com maior volume de comercialização são o mamão, laranja e banana (COSTA et al., 2015). Isso demostra as diferentes demandas destas espécies frutíferas, tendo oportunidade de inserção no mercado de hortigranjeiros. Os resultados demonstram que ainda existem espaços para a produção regional destas espécies frutíferas, visto que, a laranja, tangerina, mamão, manga e goiaba, são culturas que tem aptidão agrícola para a produção no sul do estado. 


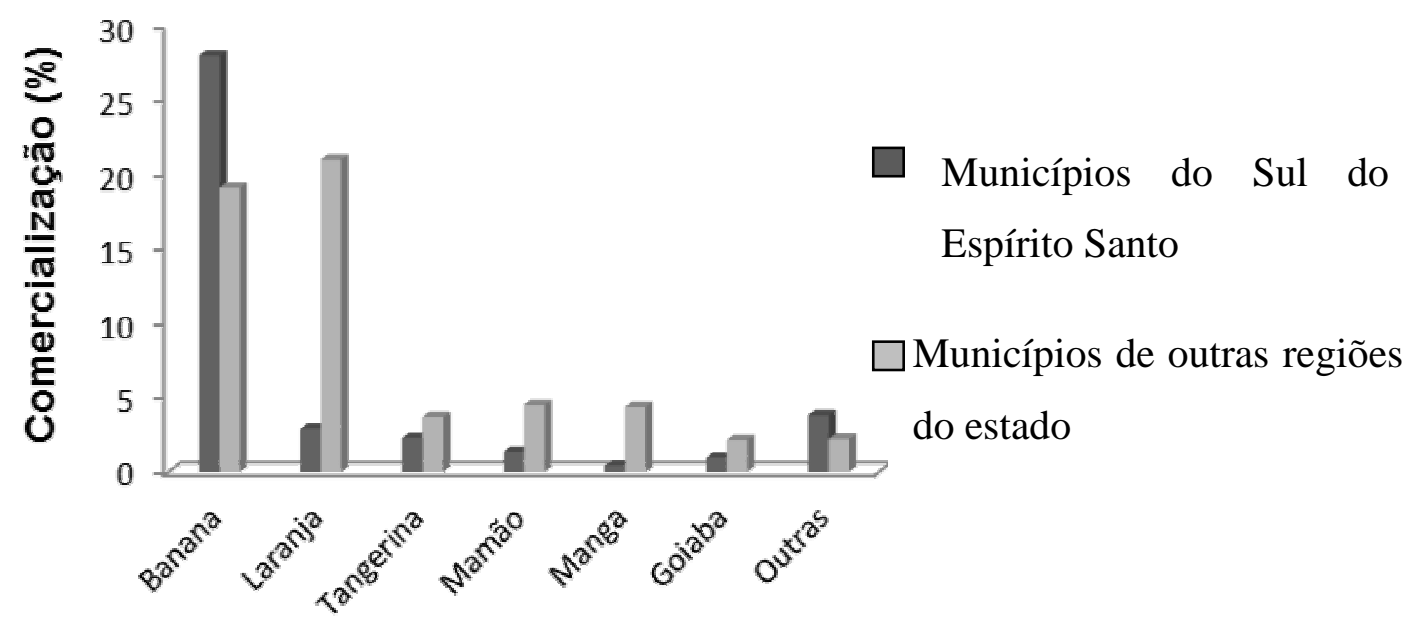

FIGURA 2 - Comercialização (\%) de frutas na Regional Sul da CEASA/ES, de acordo com procedência da produção, no ano de 2012. Cachoeiro de Itapemirim/ES, CCA/UFES.

Dentre as hortaliças de frutos, apenas o tomate e pepino foram produzidas em maior volume na região sul do estado, sendo que as demais hortaliças de fruto a maior parte do volume comercializado foram produzidas em outras regiões do estado (Figura 3). Todavia é importante ressaltar que a hortaliça de fruto com maior volume de comercialização na CEASA/ES-Sul é o tomate, assim como observado na CEASA do Rio Grande do Norte (COSTA et al., 2015). Esses resultados condizem com CUNHA \& BELIK (2012), ao destacarem a importância do tomate entre os produtos hortigranjeiros.

Observa-se uma lacuna no mercado de produtos hortigranjeiros, visto que o tomate tem grande aceitação no mercado capixaba e brasileiro, portanto o aumento do cultivo de tomate nos munícipios do Sul do estado do Espírito Santo pode ser uma ótima alternativa para os produtores desta região.

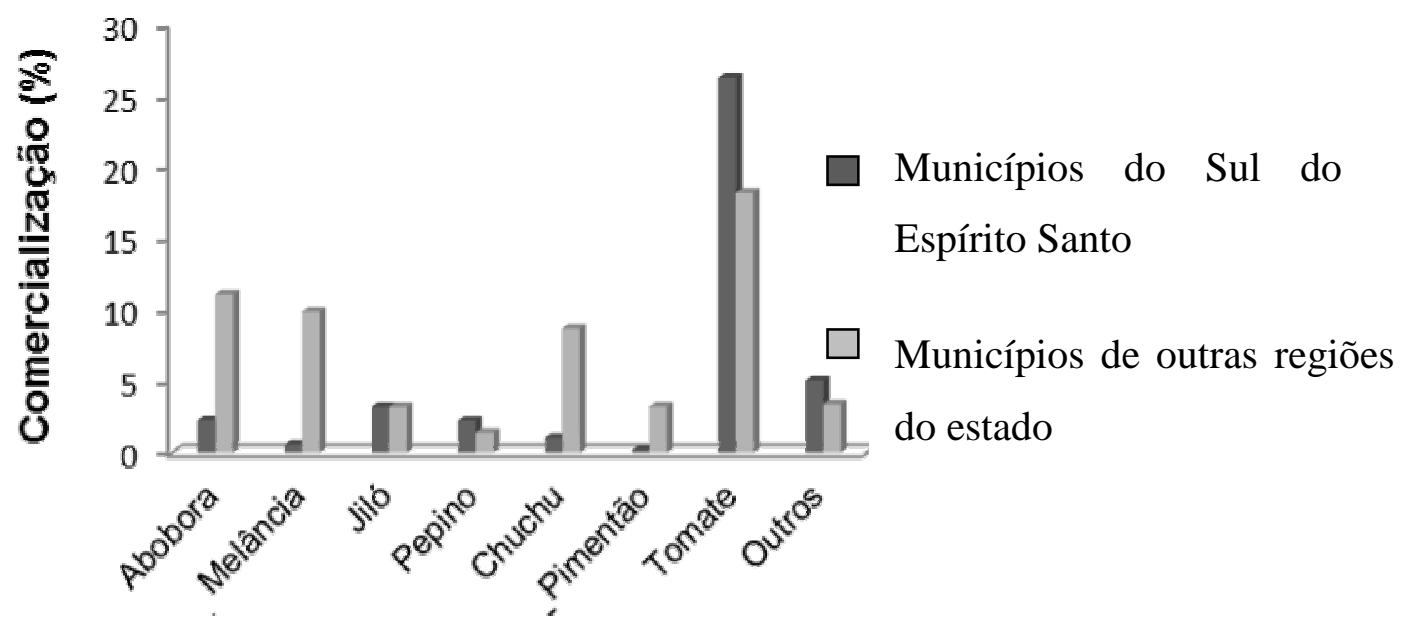

FIGURA 3 - Comercialização (\%) de hortaliças frutos na Regional Sul da CEASA/ES, de acordo com procedência da produção, no ano de 2012. Cachoeiro de Itapemirim/ES, CCA/UFES. 


\section{CONCLUSÕES}

Os principais estados que participam da comercialização de hortaliças na Regional Sul da CEASA/ES é o próprio Espírito Santo, Minas Gerais, São Paulo, Rio Grande do Sul, Bahia e Santa Catarina.

Cerca de $78,9 \%$ do volume comercializado na CEASA/ES-Sul, no ano de 2012, vieram de diferentes regiões do Espírito Santo, comprovando a importância da Regional Sul da CEASA/ES como canal de comercialização e distribuição de hortigranjeiros para o Espírito Santo, em especial a região sul do Estado

Os produtores do sul do Espírito Santo e regiões circunvizinhas, ocuparam apenas 43 e $41 \%$ do mercado para as frutas e as hortaliças de frutas no ano de 2012, respectivamente, em torno dos 30 a $38 \%$ do mercado das hortaliças de tubérculos, folhosas e raízes. Isso aponta para as demandas de mercado para esses produtos, demonstrando oportunidade de produção regional de hortifrutigranjeiros para ocupação desse mercado.

\section{AGRADECIMENTOS}

A FAPES, pelo auxílio financeiro à pesquisa e pela bolsa Pesquisador Capixaba. À UFES por toda infra-estrutura cedida. À CEASA/ES-SUL.

\section{REFERÊNCIAS}

AZEVEDO P. F.; FAULIN E. J. Distribuição de hortaliças na agricultura familiar: uma análise das transições. Informações Econômicas, v.33, n.11, p.24-37. 2003.

COSER, I.; SANTOS, T. V. dos.; BACEGA, T.; VANCIN, V. hortifrutigranjeiros: um estudo da relação consumo/produção da hortifruticultura no município de Erechim/RS. Perspectiva, Erechim. v.35, n.130, p. 35-48, junho/2011.

COSTA, A. A.; PEREIRA, M. de. G.; OLIVEIRA, S. M. da. S.; SANTOS, R. L. D. dos.; MACÊDO, C. S.; MURMANN, L. Análise do comércio de hortigranjeiros na CEASA-RN. $5^{\circ}$ Simpósio de segurança alimentar alimentação e saúde. Bento Gonçalves- RS, 2015.

CUNHA A. R. A. A. Dimensões estratégicas e dilemas das Centrais de Abastecimento. Revista de Política Agrícola (Brazil). V 15(4), p 37-46. 2006.

CUNHA A. R. A. A.; Campos J. B. O Sistema de Abastecimento Atacadista no Brasil: uma rede complexa de logística. Revista acadêmica SINAC (on line). v.4, p. 1-17. 2008.

CUNHA, A. R. A. A.; BELIK, W. A produção agrícola e a atuação das Centrais de Abastecimento no Brasil. Segurança Alimentar e Nutricional, Campinas, 19(1): 4659, 2012.

FARIA, S. G.; SOUZA, A. C. S. Análise da centralidade da CEASA Minas e, relação aos fluxos hortigranjeiros em Minas Gerais. Revista E-xacta, Belo Horizonte, v. 7, n. 2, p. 139-150, 2014.

FILGUEIRA F. A. R. Novo manual de olericultura: agrotecnologia moderna na produção e comercialização de hortaliças. Viçosa: UFV, 421p. 2008. 
PIOVESAN, A.; TEMPORINI, E. R. Pesquisa exploratória: procedimento metodológico para o estudo de fatores humanos no campo da saúde pública. Revista de Saúde Pública, Rio de Janeiro, v.29, n.4, p. 318-325, 1995.

WEGNER, R. C.; BELIK, W. Distribuição de hortifruti no Brasil: papel das Centrais de Abastecimento e dos supermercados. Cuadernos de desarrollo rural, 9 (69), 195220, 2012. 